\title{
Improving Health and Social Outcomes with Targeted Services in Comprehensive Substance Abuse Treatment
}

\author{
Dingcai Cao, Ph.D., M.S. ${ }^{1}$, Jeanne C. Marsh, Ph.D., M.S.W. ${ }^{2}$, Hee-Choon Shin, Ph.D., M.A., \\ M.P.H. ${ }^{3}$, and Christina M. Andrews, M.S.W. ${ }^{2}$ \\ ${ }^{I}$ Department of Surgery, Sections of Surgical Research and Ophthalmology \& Visual Science, University of Chicago, \\ Chicago, IL, USA, ${ }^{2}$ School of Social Service Administration, University of Chicago, Chicago, IL, USA, ${ }^{3}$ National Opinion \\ Research Center (NORC), University of Chicago, Chicago, IL, USA
}

Background: Improved life functioning along with substance use reduction is increasingly recognized as the definition of effective addiction treatment. Objectives: To assess whether targeted health and social services contribute to improved physical/mental health and employment. Methods: This study used data from the National Treatment Improvement Evaluation Study $(N=3027)$ and modeled the improvement in physical/mental health and employment at discharge or 12 months after discharge compared with intake measures as a function of receipts of matched services. Results: Receiving matched medical service improves physical health only at treatment discharge; receiving matched mental health services improves mental health at discharge and 12 months after discharge; receiving matched vocational services improves employment only 12 months after discharge. Conclusions: Need-service matching contributes to improved health and social outcomes when longitudinal assessments of treatment outcomes are used to evaluate treatment effectiveness. Scientific Significance: Study findings document the value of targeted services for achieving success in health and social functioning in comprehensive substance abuse treatment.

Keywords: need-service matching, targeted services, physical health, mental health, employment, substance abuse treatment

\section{INTRODUCTION}

Drug- and alcohol-addicted individuals often suffer from a number of other problems such as physical diseases, mental disorders, dysfunctional familial, social and financial situations. It is widely recognized that these co-occurring problems limit individual functioning and capacity to respond to treatment, and are costly to the individual, their families and society $(1,2)$. As a result, the effectiveness of substance abuse treatment is increasingly measured across multiple domains of outcome that include improvements in health and social functioning (2).

Comprehensive substance abuse treatment - where health and social services are provided along with substance abuse treatment - is a treatment approach specifically designed to improve health and social functioning. Evidence indicates that substance abuse treatment patients who receive comprehensive services show improved outcomes not only in terms of reduced posttreatment substance abuse but also improved functioning (3-6).

Increasingly, models of comprehensive substance abuse treatment have specific components of services that contribute to improved outcomes. Such studies conceptualize specific health and social services, including substance abuse services, as having an impact on a range of substance use, as well as health and social outcomes. For example, studies have shown that linkage or access services, including services like transportation, child care, and outreach, increase the likelihood that a patient will be able to reach or obtain services and ultimately reduce their post-treatment substance use $(7,8)$. Evidence also indicates HIV programming offered as part of comprehensive substance abuse treatment reduces risk behaviors related to the development of HIV (9). Finally, research shows that when health and social services are matched to specific patient-identified needs, comprehensive services can be effectively delivered as part of substance abuse treatment (10-14).

Although the evidence is substantial that need-service matching is a key component in the delivery of comprehensive substance abuse treatment, most studies have

Address correspondence to: Jeanne C. Marsh, School of Social Service Administration, University of Chicago, 969 E. Sixtieth Street, Chicago, IL 60637, USA. Tel: +773-702-1144. Fax: +773-702-0874. E-mail: jmarsh@uchicago.edu 
examined the impact of matching on substance abuse outcomes. As a result, we have limited knowledge of the impact of need-service matching on health and social service outcomes. The question remains: If health and social services are targeted to patients who have identified needs in specific areas of health and social functioning, will they show subsequent improvements in those areas of functioning?

A final question of concern for treatment effectiveness researchers has been the durability of effects, that is, when changes resulting from treatment can be expected and how long they can be expected to last. Increasingly, some forms of addiction are considered to be chronic, with the remediation necessarily monitored over time $(1,15)$. The preponderance of treatment effectiveness studies have measured outcome at discharge and 6-12 months post-discharge, but the impact of treatment on various other outcome measures rarely has been compared over time (4).

Research in the past 30 years has documented the effectiveness of addiction treatment primarily in terms of reductions in substance use and less frequently in terms of improvements in health and social functioning. It has additionally shown that targeted services, those services matched to patient needs in a specific area of health and social functioning, will contribute to improvements in functioning. Despite these advances, we have little knowledge of the type of durability of change that can be achieved. The purpose of this study is, therefore, threefold: (1) to examine improvements in addiction-related health and social outcomes, specifically, outcomes of physical health, mental health, and employment; (2) to evaluate whether targeting services to improve physical health, mental health and employment during substance abuse treatment is related to outcomes in those areas; and (3) to assess improvements at two time points of discharge and 12 months post-discharge. The study is designed to increase understanding of how health and social services targeted to specific domains of treatment outcomes can foster improvement in those areas in order to contribute to knowledge on the development of more effective services.

\section{METHODS}

\section{Design and Sample}

The National Treatment Improvement Evaluation Study (NTIES) data are a national, longitudinal follow-up study of the impact of drug and alcohol treatment programs receiving funding from the Substance Abuse and Mental Health Services Administration, Center for Substance Abuse Treatment (CSAT). The study was designed to assess treatment effectiveness and improvement in treatment services supported by CSAT. A focus of this study was the extent to which a comprehensive treatment model could be validated - that is, the extent to which more comprehensive treatment yields more effective results in terms of patient access to services and outcomes of treatment (16). To support inquiry into this question, patient and service data were collected from patient interviews obtained at treatment intake, treatment exit, and 12 months after treatment exit. Organizational data were collected from interviews with treatment program administrators at two points in time during a 12-month period. The details of the design and sampling procedures of NTIES are available elsewhere (16).

NTIES employed a two-stage sampling design: the first stage samples treatment providers or service delivery units, and the second stage samples patients within the selected providers. Non-response occurred at the first and second stages due to non-cooperation of the sample providers and the problems of locating and obtaining interviews from sampled patients selected from cooperating providers. Of 7782 NTIES eligible patients, 85\% (6593 patients) completed the intake interview. All of the 6593 patients who completed the intake interview were targeted for treatment discharge and follow-up interviews about 12 months after leaving treatment. The response rate for the discharge interview was $80 \%$, and the 12 month follow-up response rate was $82 \%$ (slightly higher when the small number of deceased and other excluded cases are removed from the denominator) (16). As a result of the two stage sampling design, where clients were selected in the study with probability sampling at the second stage, they are representative only of clients entering programs funded by the Center for Substance Abuse Treatment during a specific period.

The analytic sample for this secondary analysis was a subset of 4526 patients who completed all intake, treatment discharge, and follow-up interviews. After excluding patients from correctional facilities $(N=$ $1384)$ and those who reported no need $(n=115)$, the final analytic sample consisted of 3027 patients from 59 service delivery units (1105 female and 1922 male patients).

\section{Measures}

Treatment Outcomes: Physical Health, Mental Health, and Employment

Improvements in physical health, mental health, and employment were measured based on a patient's responses to questionnaire items administrated at intake, at discharge, and approximately 12 months after discharge from treatment.

Physical health was measured by self-rated general health at intake, discharge, and post-discharge. Based on a patient's response to the item "in general, was your health excellent, good, fair or poor?," we constructed a dichotomized variable to indicate whether his/her physical health status at discharge or post-discharge was improved (health rating at discharge or post-discharge was better than that at intake) or not (health rating at discharge or post-discharge was the same as or worse than health rating at intake).

To assess mental health status at intake, at discharge, and 12 months post-discharge, we used responses on questionnaire items related to mental health symptoms. The approach was similar to the earlier work of NTIES 
researchers (16) except that we did not include measures of utilization of mental health services. Different numbers of mental health symptoms were assessed at different time points, with the same four types of symptoms (suicide attempts, bothered by mental health, panic, and depression) measured at intake and 12 months postdischarge, while only two symptoms (suicide attempts and depression) were measured at discharge. The four mental health symptoms measured at intake and 12 months post-discharge included items designed to assess mental health symptoms that were independent of the effects of drug use. Specifically, they included suicide attempts, being troubled or bothered by mental health, symptoms of panic disorder that was not induced by drug (sudden fear in situations most others would not have been afraid with symptoms of trembling, sweating, or troubling breathing), and symptoms of depression not induced by drug (feeling very sad or depressed and lost interest in things you cared about for 2 weeks). The Cronbach alpha of the items measuring mental health symptoms was .84 at intake (11 items) and .83 (12 items) at 12 months post-discharge, indicating good internal consistency of the items. Three items measuring symptoms of suicide attempts and depression at discharge had a Cronbach alpha of .55. The relatively low Cronbach alpha value for the items at discharge reflects the small number of items that were used since Cronbach alpha depends not only on the correlations among items but also on the number of items. Mental health at discharge was considered to be improved if the number of symptoms (suicide attempts and depression) at discharge was fewer than that at intake. Twelve-month post-discharge mental health was considered improved if the number of mental health symptoms (suicide attempts, bothered by mental health, panic, and depression) was reduced compared to that at intake.

Employment was measured as whether a patient was employed at intake, at discharge, and post-discharge. Based on the patients' responses to the item "did you have a job," we created a dichotomized variable to indicate whether employment status was improved at discharge or post-discharge (from being unemployed at intake to being employed at discharge or post-discharge).

To insure that the dichotomized outcomes (improved or not) did not result in the loss of ability to detect significant relationships that would have been found when using the continuous form of the variables, we attempted two additional modeling strategies. The first strategy used the post-treatment measures (at discharge or 12 months after discharge) as the outcome variables while using the intake measures as the control variables. The second strategy used the changes in ratings between posttreatment and intake on the measures as the outcome variables. Based on patients' intake and post-treatment rating, we constructed a variable for physical health status change as deteriorating, same, or improved. For mental health, the change score in mental health severity index was the difference between the pre-treatment mental health severity and post-treatment mental health severity. For employment, we created a four-category variable to indicate employment status change at the intake and post-treatment measures: (1) unemployed (at intake)unemployed (at discharge or 12 months after discharge), (2) unemployed-employed, (3) employed-unemployed, and (4) employed-employed. These two additional modeling strategies essentially yielded results comparable to the one with the dichotomized outcome variables. To facilitate ease of interpretation, we reported the results only from the dichotomized outcomes.

\section{Matched Service and Control Variables}

The impact of need-service matching on health and social outcomes was evaluated by including a measure of matched services that was based on service needs reported at intake and service receipt reported at discharge. Following our previous work $(6,9,14,17)$, we created seven types of service including (1) medical services (health services, AIDS prevention services, medical pregnancy services), (2) mental health services (mental health counseling or treatment), (3) family and life skills services (parenting, domestic violence counseling, family services, assertiveness training, life skills, familyplanning, non-medical pregnancy services), (4) vocational, (5) housing, (6) financial services (help collecting benefits, help getting alimony/child support), and (7) substance abuse counseling services (including drug/alcohol counseling, 12-step meetings, drug prescription for alcohol/drug problems). Service need was measured as a response to a question in the intake questionnaire as to "how important" ("very," "somewhat," "not at all"; with "very" or "somewhat") the patient considered the service to be. Service receipt was measured using an item on the discharge questionnaire that asked whether the patient had received service in each of these areas. Targeted or matched service was a binary variable constructed to indicate whether a patient reported receiving a particular service during treatment that they had reported needing at intake. The matched service variable had value of 1 if a patient received a service that he or she needed, while the value was 0 if a patient did not need a service or a patient needed a service but did not receive it. Although dichotomized service variables led to less statistical power, this approach was justified due to their skewed distribution (18).

Control variables were measured at the patient and organizational levels. Patient-level control variables included age in years, race/ethnicity (non-Hispanic whites, non-Hispanic blacks, or Hispanics, with whites as the reference category), education in years, pre-treatment health status (whether health limited their work), family violence (whether they had ever been beaten by a spouse or partner), source of payment for services (private, public, or uninsured), previous alcohol or drug treatment experience, pre-treatment drug/alcohol use (measured as the number of days in the past 30 days using the five most frequently used substances, including alcohol, marijuana, cocaine, cocaine power, and heroin) to quantify substance abuse severity, and treatment duration in weeks. Organizational-level control variables, 
derived from administrative interviews, included JCAHO (currently known as The Joint Commission) accreditation, modality (non-methadone outpatient, methadone, short-term residential, or long-term residential, with nonmethadone outpatient as the reference category), ownership (private vs. public with public program as the reference category), onsite service availability (measured as the number of onsite services provided by the program, including academic training, vocational training, medical, psychiatric, or pregnancy services), and frequency of counseling. Additional details about the construction of matched service and control variables are described elsewhere $(6,9,14,17)$.

\section{Statistical Analysis}

In the NTIES data set, $0-15 \%$ of data were missing (except for the accreditation variables where up to $60 \%$ of data were missing for African Americans). Since data missing for key variables (as described in previous work (16)), did not appear to be missing systematically, a multiple imputation procedure (19) that assumed the data were missing at random was conducted to fill in the missing values (20). In the multiple imputation procedure, each missing value was replaced with five plausible values using Markov Chain Monte Carlo method. Imputation was conducted for the organizational variables and patient-level variables independently. The resulting five imputed datasets for both organizational-level and patient-level data were merged into five datasets for further univariate and multivariate analysis. The statistical outputs from the five datasets were combined to evaluate the effects of matched services on improved physical health, mental health, or employment.

Separate analyses were conducted to assess the impact of targeted services over time. Analyzing the data separately at discharge and 12 months post-discharge was appropriate given the time periods between entry and discharge, and discharge and 12 months post-discharge were not consistent for each patient. Patients spent an average of 14 weeks in treatment, that is, between entry and discharge, but the specific amount of time differed for each patient. Further, while the period between discharge and 12 months post-discharge was approximately 12 months, it was different for every patient. Thus, it was not possible to assume a consistent interval between measurements at discharge and 12 months post-discharge. Additionally, for measures of mental health status, different items were used at different time points.

For the univariate analysis, we used a logistic regression to investigate the association between an outcome at discharge or 12 months post-discharge (improved physical health, mental health, or employment) and each of the matched services (medical, mental health, family/life skills, vocational, housing, financial, and substance abuse counseling). For each outcome at discharge or 12 months post-discharge, a multivariate analysis controlled for patient and organizational characteristics by using a generalized linear mixed model with a logit link function to account for potential correlation within service delivery units. Results are reported here using estimated odds ratios, and $95 \%$ confidence intervals for matched service.

All analyses, including descriptive comparison, multiple imputation of missing values, and generalized linear mixed modeling were conducted in SAS 9.1.3.

\section{RESULTS}

Compared with measures at intake, the respective proportions of patients reporting improved outcomes at discharge and post-discharge were $20.2 \%$ and $23.0 \%$ for physical health, $51.9 \%$ and $54.3 \%$ for mental health, and $11.2 \%$ and $27.2 \%$ for employment. Table 1 shows the odds ratios of improved outcomes between patients who received matched services and those who did not received matched services. The first column in Table 1 shows the percentage of patients who received matched services in seven specific areas: medical, mental health, family, vocational, housing, financial, and substance abuse counseling. Overall, the majority of patients $(94.6 \%)$ received matched substance abuse counseling service, while nearly $40 \%$ of patients received matched medical or family services. For other service categories (mental health, vocational service, housing, and financial services), the proportion of patients who received matched services was relatively low (between $13.8 \%$ and $21.0 \%$ ), even though over $60 \%$ of patients reported needs in each of these service categories (13).

The second column indicates the measurement time for the outcomes. The last three columns of Table 1 list the univariate analytic results for examining the association between receiving matched services and improvements in physical health (the fourth column), mental health (the fifth column), and employment (the sixth column), respectively. For each outcome, the proportion of improved outcomes at each time point among the patients who received matched service and odd ratios (95\% confidence intervals, CI) comparing improvement in the outcomes between patients who received matched services and those who did not are reported. A significant odds ratio larger than 1 (as the confidence interval does not included 1.0) indicates that receiving matched services is associated with a higher odds of improvement in outcome; while a significant odds ratio less than 1 indicates that receiving matched services is associated with a lower odds of improvement in outcome. The univariate analysis results indicates that, for physical health, none of the matched services was associated with improved physical health at discharge, while matched medical service $(\mathrm{OR}=.789,95 \% \mathrm{CI}=.661-.942)$, mental health service $(\mathrm{OR}=.737,95 \% \mathrm{CI}=.587-.92)$, and financial service $(\mathrm{OR}=.743,95 \% \mathrm{CI}=.57-.961)$ were associated with lower odds of improvement at 12 months post-discharge. In contrast, for mental health, all of the matched service areas except substance/alcohol abuse counseling were significantly associated with higher odds 


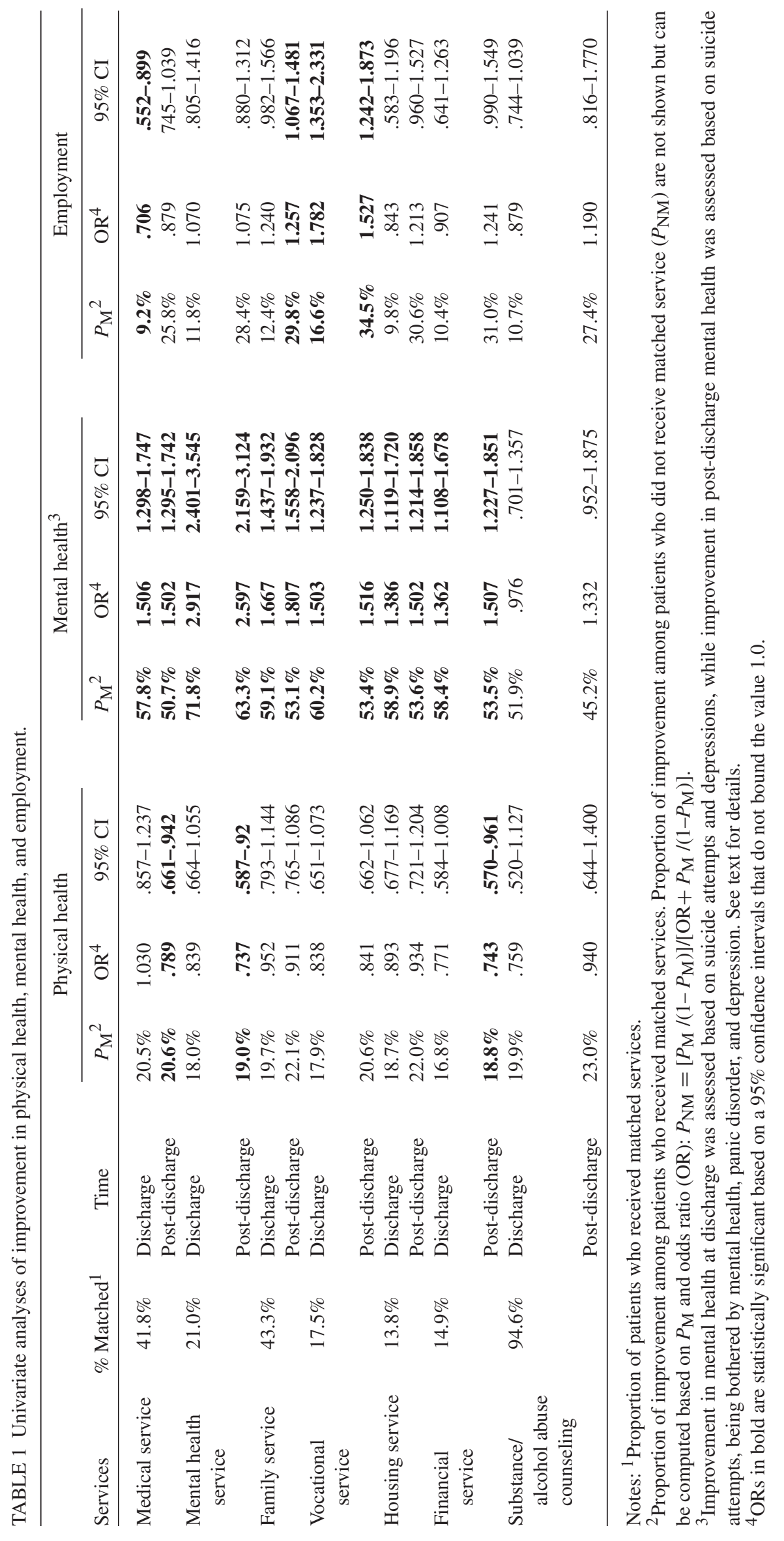


of improvement at both discharge and 12 months postdischarge (at discharge: all ORs between 1.362 and 2.917 are significant; at post-discharge: all ORs between 1.502 and 2.597 are significant). Finally, for employment, at discharge, matched medical services $(\mathrm{OR}=$ $.706,95 \% \mathrm{CI}=.552-.899)$ were associated with lower odds of improvement but matched vocational services $(\mathrm{OR}=1.782,95 \% \mathrm{CI}=1.353-2.331)$ were associated with higher odds of improvement in employment. For 12month post-discharge, employment, matched family service, and vocational service were associated with higher odds of improvement (all ORs between 1.257 and 1.527 are significant).

Table 2 shows that after controlling patient and organizational characteristics, matched medical services significantly increased the odds of improvement in physical health at discharge $(\mathrm{OR}=1.242,95 \% \mathrm{CI}=1.005-1.536)$ but none of the matched services was significantly associated with improvement in physical health at postdischarge. For mental health, matched medical health service and mental health service increased the odds of improvement in mental health at discharge (medical health service: $\mathrm{OR}=1.287,95 \% \mathrm{CI}=1.075-1.541$; mental health service: $\mathrm{OR}=2.074,95 \% \mathrm{CI}=1.663-$ 2.587); while matched mental health service and family service remained significant predictors of improved mental health (mental health service: $\mathrm{OR}=1.890,95 \% \mathrm{CI}$ $=1.537-2.324$; family service: $\mathrm{OR}=1.384,95 \% \mathrm{CI}$ $=1.153-1.660)$. Finally, for employment at discharge, none of the matched services were related to improvement at discharge. For employment at post-discharge, matched family service $(\mathrm{OR}=1.254,95 \% \mathrm{CI}=1.021-$ $1.539)$ and vocational service $(\mathrm{OR}=1.342,95 \% \mathrm{CI}=$ $1.044-1.1 .724)$ predicted higher odds of improvement in employment but matched medical service $(\mathrm{OR}=.801$, $95 \% \mathrm{CI}=.657-.976)$ predicted lower odds of improvement in employment.

\section{DISCUSSION}

In this study, we evaluated the effectiveness of targeted services for physical health, mental health, and employment outcomes right after treatment completion and during the follow-up period. Our analyses suggest

TABLE 2. Multivariate analyses of improvement in physical health, mental health, and employment.

\begin{tabular}{|c|c|c|c|c|c|c|}
\hline \multirow[b]{2}{*}{ Matched service } & \multicolumn{2}{|c|}{ Physical health ${ }^{1}$} & \multicolumn{2}{|c|}{ Mental health $^{2}$} & \multicolumn{2}{|c|}{ Employment $^{3}$} \\
\hline & $\mathrm{OR}^{4}$ & $95 \% \mathrm{CI}$ & $\mathrm{OR}^{4}$ & $95 \% \mathrm{CI}$ & $\mathrm{OR}^{4}$ & $95 \% \mathrm{CI}$ \\
\hline \multicolumn{7}{|l|}{ Discharge } \\
\hline Medical service & 1.242 & $1.005-1.536$ & 1.287 & $1.075-1.541$ & .773 & $.570-1.049$ \\
\hline Mental health service & .918 & $.709-1.190$ & 2.074 & $1.663-2.587$ & 1.026 & $.718-1.464$ \\
\hline Family service & .975 & $.770-1.235$ & 1.193 & $.985-1.445$ & 1.255 & $.931-1.690$ \\
\hline Vocational service & .818 & $.615-1.089$ & 1.163 & $.915-1.478$ & 1.404 & $.987-1.997$ \\
\hline Housing service & 1.029 & $.760-1.392$ & 1.018 & $.789-1.314$ & .818 & $.528-1.267$ \\
\hline Financial service & .752 & $.547-1.033$ & .915 & $.702-1.193$ & 1.243 & $.798-1.935$ \\
\hline Substance/alcohol abuse counseling & .853 & $.481-1.513$ & 1.039 & $.637-1.696$ & 1.050 & $.457-2.414$ \\
\hline \multicolumn{7}{|l|}{ Post-discharge } \\
\hline Medical service & .923 & $.753-1.132$ & 1.177 & $.987-1.403$ & .801 & $.657-.976$ \\
\hline Mental health service & .857 & $.666-1.103$ & 1.890 & $1.537-2.324$ & .956 & $.760-1.203$ \\
\hline Family service & 1.050 & $.837-1.316$ & 1.384 & $1.153-1.660$ & 1.254 & $1.021-1.539$ \\
\hline Vocational service & .915 & $.697-1.202$ & 1.217 & $.965-1.535$ & 1.342 & $1.044-1.724$ \\
\hline Housing service & 1.104 & $.830-1.469$ & 1.023 & $.801-1.308$ & 1.187 & $.909-1.551$ \\
\hline Financial service & .804 & $.593-1.089$ & 1.109 & $.858-1.433$ & .860 & $.646-1.145$ \\
\hline Substance/alcohol abuse counseling & 1.002 & $.490-2.046$ & 1.107 & $.747-1.643$ & 1.372 & $.636-2.958$ \\
\hline
\end{tabular}

Notes: ${ }^{1}$ For improvement in physical health at discharge, significant control variables included age $(\mathrm{OR}=.986,95 \% \mathrm{CI}=.973-1.000, p=$ $.045)$ and health-limited work $(\mathrm{OR}=.699,95 \% \mathrm{CI}=.556-.880, p=.004)$; for improvement in physical health at post-discharge, significant control variables included Hispanic $(\mathrm{OR}=1.461,95 \% \mathrm{CI}=1.060-2.016, p=.023)$, pre-treatment health-limited work $(\mathrm{OR}=.629,95 \% \mathrm{CI}$ $=.504-.786, p<.001)$, and methadone treatment program $(\mathrm{OR}=2.154,95 \% \mathrm{CI}=1.401-3.312, p=.001)$.

${ }^{2}$ For improvement in mental health at discharge, significant control variables included male gender $(\mathrm{OR}=.728,95 \% \mathrm{CI}=.588-.901, p$ $=.005)$, non-Hispanic black $(\mathrm{OR}=.744,95 \% \mathrm{CI}=.591-.936, p=.014)$, health-limited work $(\mathrm{OR}=1.349,95 \% \mathrm{CI}=1.113-1.635, p=$ $.004)$, pre-treatment mental health visit $(\mathrm{OR}=1.450,95 \% \mathrm{CI}=1.292-2.626, p<.001)$. For improvement in mental health at post-discharge, significant control variables included African American $(\mathrm{OR}=.731,95 \% \mathrm{CI}=.588-.909, p=.007)$, non-Hispanic black $(\mathrm{OR}=.731,95 \% \mathrm{CI}$ $=.588-.909, p=.007)$, health-limited work $(\mathrm{OR}=1.276,95 \% \mathrm{CI}=1.058-1.540, p=.013)$, pre-treatment mental health visit $(\mathrm{OR}=1.127$, $95 \% \mathrm{CI}=1.024-1.240, p=.017)$, short-term residential program $(\mathrm{OR}=1.668,95 \% \mathrm{CI}=1.061-2.622, p=.028)$, methadone treatment program $(\mathrm{OR}=.502,95 \% \mathrm{CI}=.324-.778, p=.003)$, and onsite service availability $(\mathrm{OR}=1.116,95 \% \mathrm{CI}=1.001-1.246, p=.049)$.

${ }^{3}$ For improvement in employment at discharge, significant control variables included male gender $(\mathrm{OR}=1.499,95 \% \mathrm{CI}=1.036-2.170, p$ $=.033)$, pre-treatment drug use $(\mathrm{OR}=.989,95 \% \mathrm{CI}=.979-.999, p=.035)$, treatment duration $(\mathrm{OR}=1.033,95 \% \mathrm{CI}=1.022-1.044, p<$ $.001)$, short-term residential program $(\mathrm{OR}=.135,95 \% \mathrm{CI}=.033-.545, p=.007)$, and long-term residential program $(\mathrm{OR}=.150,95 \% \mathrm{CI}$ $=.053-.423, p=.001)$. For improvement in employment at post-discharge, significant control variables included male gender $(\mathrm{OR}=1.394$, $95 \% \mathrm{CI}=1.100-1.767, p=.008)$ and education $(\mathrm{OR}=1.072,95 \% \mathrm{CI}=1.022-1.124, p=.006)$.

${ }^{4} \mathrm{ORs}$ in bold are statistically significant based on a $95 \%$ confidence intervals that do not bound the value 1.0. 
that while all of the targeted services examined were related to outcome, the timing of the observed effect at discharge or 12 months post-discharge - depended on the outcome. In the sample we used, medical services helped to improve physical health, but only at discharge. Receiving matched mental health service improved mental health both at discharge and post-discharge, while receiving matched vocational service improved employment only at post-discharge. These findings indicate not only that targeted services help to improve specific health and social outcomes but also that the benefit of such services may be manifested at different time points after treatment. As such, these finding are congruent with the growing consensus in the substance abuse treatment field regarding the need to examine the course of treatment outcomes longitudinally (21).

In our sample, a large percentage of patients, $41.8 \%$, received targeted or matched physical health services. This finding is similar to studies by Hser et al. (22) and Smith and Marsh (8), which reported rates of receipt of targeted physical health services of $44 \%$ and $29 \%$, respectively. Physical health services received during substance abuse treatment affected health status outcome at discharge, but not at 12 months post-discharge. In fact, the univariate analysis (Table 1) indicated receiving matched medical service was negatively associated with long-term health status. This finding, while perhaps somewhat puzzling, could be related to the "need" aspect of targeted services. In this sample, patients who reported a need for medical services also reported worse physical health at entry to treatment. As such, they may also be more likely to return to poor health status once they no longer have access to medical services for which they reported a need. These findings suggest that among those reporting health problems, gains in health status made during treatment will not necessarily be sustained without continued access to medical care after discharge.

Receiving matched mental health services was associated with improved mental health status not only at discharge but also at 12 months post-discharge, suggesting that targeting mental health services as a component of substance abuse treatment for individuals with cooccurring psychiatric problems can have a sustained, beneficial effect on mental health. There are at least a couple of reasons for the observed relationship. First, through receipt of mental health services, patients may have learned coping strategies to help reduce mental health symptoms that they are able to maintain over time. Moreover, patients may have addressed social and other environmental problems during the course of receiving mental health services. Improvements in their environmental context, when sustained after the conclusion treatment, could support the durability of gains in mental health status over time. Given the high rate of co-occurring mental health problems among individuals in substance abuse treatment $(23,24)$, it is useful to know that targeted mental health services can have a significant and durable effect.

The findings indicate that receipt of medical services was also associated with improved mental health at discharge. This finding is congruent with a broad body of literature documenting a significant positive relationship between physical health and mental health status (25). Yet, similar to the effect for physical health, when medical services are terminated at the conclusion of treatment, the effect is not sustained. Additionally, receipt of matched family services was associated with mental health status at 12 months post-discharge, suggesting that among patients struggling with family issues, receipt of family-focused counseling and skills training helped them to improve their psychological well-being. It is possible that skills learned via these services - including parenting training, family-planning, and life skills training - may take time to be fully implemented and realized.

Finally, throughout the treatment literature, employment is understood to be a facilitator of recovery as well as a deterrent against relapse (26). Findings from this study indicate that matched vocational services were not associated with improved work status at discharge, but were associated with improved work status at 12 months post-discharge. There are several possible reasons that the impact of matching vocational services was manifest only a year after the conclusion of treatment. First, a significant proportion of patients who received matching employment services received some level of skills training. Patients may have needed to complete this training before seeking job opportunities. Second, many patients may need to address primary substance abuse issues before addressing ancillary concerns such as employment, leading to lag time in the start of employment preparation and searching. This may be particularly true for those patients with more severe substance abuse and mental health problems. Finally, if the patient perceives improvement in vocational status as the attainment of paid employment, the benefit of vocational services may not become apparent for several months, as securing employment can take a substantial amount of time.

This finding is congruent with prior research examining the effect of providing vocational services to those patients reporting a need for them. Hser et al. (22) found that at 6 months post-discharge, patients who needed and received employment services showed significantly greater improvement in the employment scale of the Addiction Severity Index than other patients. However, these findings are inconsistent with at least one previous analysis - a discrepancy that may be explained by measurement differences. Using the same dataset, Dunlap et al. (27) found that no service variables were related to post-discharge employment in outpatient drug-free treatment programs in the NTIES sample. However, their analysis used a simple measure of service receipt. In fact, when we used the same measure of service receipt, we replicated Dunlap et al.'s null finding. In the present study, using a measure of matched services, we found that receiving matched vocational services contributed to improved employment at 12 months post-discharge. This finding highlights the fact that a measure of services that considers whether patients need the service is more 
predictive of treatment outcome than measures that do not, at least in the case of employment.

Moreover, this study also found that matched medical and family services were also related to employment at 12 months post-discharge. In the case of medical services, receipt of matched services was associated with less improvement in employment. As mentioned above, this may be the result of differences in health status among those who reported a need for medical services. Patients who needed and received medical services due to poor health, but stopped receiving them due to the conclusion of treatment, may have experienced returning health problems as a barrier to obtaining employment. Matched family services, including family counseling, family-planning services, and life skills training, were also associated with improved employment status at 12 months post-discharge. Such services may have assisted patients struggling with family problems to address and better manage family issues, thus enabling them to focus more attention on other goals such as identifying employment.

Findings from this study provide additional insights regarding the impact and durability of targeted services on health and social outcomes. Targeted services had an impact on all three outcomes of interest: physical health, mental health, and employment. The impact on physical health was significant at discharge, but the effects were not sustained 12 months later, while the impact on mental health was significant at both discharge and 12 months post-discharge. We conjecture that health deteriorates in the absence of immediately available medical services that are part of substance abuse treatment while the mental health services provided, which included counseling and skills training, may result in the acquisition of knowledge and skills that patients could use to maintain reduced mental health symptomotology. Finally, the impact of targeted services on employment was not evident until 12 months post-discharge. Considering that the sample included a number of patients in residential treatment who were not working at the time of discharge, it would be expected that evidence of the impact of matched vocational services would most likely be found at 12 months post-discharge. Overall, the findings indicate the value of measuring the impact of substance abuse treatment on health and social outcomes over time to increase understanding of the timing and durability of treatment effects.

The study has several limitations. First, the sample selection procedure used for the NTIES data introduce two potential sources of bias that limit the generalizability of NTIES - patients' self-selection into the treatment and non-responses to the interview. In the first case, it was not possible to evaluate the extent to which study participants and non-participants differed. In the case of response bias, some level of non-response is expected from any large-scale national survey. However, analyses of selection and non-response bias conducted by Gerstein and colleagues (28), who compared the NTIES response rate to other large-scale follow-up studies, indicated the bias in the NTIES sample is limited. Second, the NTIES sample consists of treatment programs receiving competitive demonstration funds from the CSAT with the specific aim to develop more comprehensive service programs. It is therefore possible that observed patterns are not generalizable to other substance abuse treatment programs. Third, the survey relied on self-reported measures of services received and targeted outcomes in the areas of physical health, mental health, and employment. It is possible that patients may not have accurately remembered what services they received, or were unable to identify what type of service they received. The lack of in-depth measurements of these variables may not be sufficient to assess the effects of services on treatment outcomes (27). Finally, NTIES data were collected between 1993 and 1995 and became publicly available in 1997. Available evidence suggests that service delivery patterns have changed in the past decade, and health and social services are less frequently available in substance abuse treatment (12). However, the data remains relevant for our research purposes since the relations among mechanisms of service delivery and treatment outcome are unlikely to change over time.

In sum, results of the study contribute valuable information for the development of effective substance abuse treatment services by documenting the impact of targeted services on health, mental health, and employment outcomes. Improved health and social outcomes, as well as reductions in substance use, are important measures of treatment success. Study findings document the value of targeted services for achieving success in health and social functioning as part of comprehensive substance abuse treatment.

\section{ACKNOWLEDGEMENTS}

This research was supported by the National Institute on Drug Abuse grant: R01DA018741.

\section{Declaration of Interest}

The authors report no conflicts of interest. The authors alone are responsible for the content and writing of this article.

\section{REFERENCES}

1. McLellan AT, McKay JR. Components of successful addiction treatment: What does research say? In Principles of Addiction Medicine. Graham AW, Schultz T, eds. (2nd ed.). Chicago, IL: University of Chicago Press, 1998; 24-48.

2. McLellan AT, McKay JR, Forman R, Cacciola J, Kemp J. Reconsidering the evaluation of addiction treatment: From retrospective follow-up to concurrent recovery monitoring. Addiction 2005; 100:447-458.

3. McLellan AT, Arndt IO, Metzger DS, Woody GE, O'Brien CP. The effects of psychosocial services in substance abuse treatment. J Am Med Assoc 1993; 269(15):1953-1959. 
4. Hubbard RL, Craddock SG, Anderson J. Overview of 5year followup outcomes in the drug abuse treatment outcome studies (DATOS). J Subst Abuse Treat 2003; 25(3): 125-134.

5. McLellan AT, Hagan TA, Levine M, Gould F, Meyers K, Bencivengo M, Durell J. Supplemental social services improve outcomes in public addiction treatment. Addiction 1998; 93(10):1489-1499.

6. Marsh JC, Cao D, D'Aunno T. Gender differences in the impact of comprehensive services in substance abuse treatment. J Subst Abuse Treat 2004; 27(4):289-300.

7. Friedmann PD, D‘Aunno TA, Jin L, Alexander JA. Medical and psychosocial services in drug abuse treatment: Do stronger linkages promote client utilization? Health Serv Res 2000; 35(2):443-465.

8. Smith BD, Marsh JC. Client-service matching in substance abuse treatment for women with children. J Subst Abuse Treat 2002; 22(3):161.

9. Cao D, Marsh JC, Shin HC. Gender and racial/ethnic disparities in the impact of HIV prevention programming in substance abuse treatment. Am J Drug Alcohol Abuse 2008;34:730-740.

10. Hser Y.-I, Grella CE, Hsieh S-C, Anglin MD, Brown BS. Prior treatment experience related to process and outcomes in DATOS. Drug Alcohol Depend 1999; 57(2): 137-150.

11. Friedmann PD, Hendrickson JC, Gerstein DR, Zhang Z. The effect of matching comprehensive services to patients' needs on drug use improvement in addiction treatment. Addiction 2004; 99(8):962-972.

12. Campbell CI, Wells R, Alexander JA, Jiang L, Nahra TA, Lemak CH. Tailoring of outpatient substance abuse treatment to women, 1995-2005. Med care 2007; 45(8):775.

13. Marsh JC, Cao D, Guerrero E, Shin HC. Closing the needservice gap: Gender differences in matching services to client needs in comprehensive substance abuse treatment. Soc Work Res 2009; 33(3):183-192.

14. Marsh JC, Cao D, Guerrero E, Shin HC. Need-service matching in substance abuse treatment: Racial/ethnic differences. Eval Program Plann 2009; 32(1):43-51.

15. McLellan AT, Lewis DC, O`Brien CP, Kleber HD. Drug dependence, a chronic medical illness: Implications for treatment, insurance, and outcomes evaluation. J Am Med Assoc 2000; 284(13):1689-1695.
16. Gerstein DR, Datta AR, Ingels JS, Johnson RA, Rasinski KA, Phillips DB, Anderson DW, Condelli WG, Collins JS. Final Report. NTIES: National Treatment Improvement Evaluation Study. Rockville, MD: Center for Substance Abuse Treatment, Substance Abuse and Mental Health Services Administration, 1997.

17. Marsh JC, Shin HC, Cao D. Gender differences in clientprovider relationship as active ingredient in substance abuse treatment. Eval Program Plann 2010; 33:81-90.

18. MacCallum RC, Zhang S, Preacher KJ, Rucker DD. On the practice of dichotomization of quantitative variables. Psychol Methods 2002; 7(1):19-40.

19. Rubin DB. Multiple Imputation for Non-Response in Surveys. New York: Wiley, 1987.

20. Little RJ, Rubin DB. Statistical Analysis with Missing Data. New York: Wiley, 1987.

21. Hser YI, Teruya C. Introduction to the special issues on longitudinal research on substance abuse and health services. Evaluation Rev 2007; 31(6):511-514.

22. Hser Y-I, Polinsky ML, Maglione M, Anglin MD. Matching clients' needs with drug treatment services. J Subst Abuse Treat 1999; 16(4):299-305.

23. Kessler RC, Nelson CB, McGonagle KA, Edlund MJ, Frank RG, Leaf PJ. The epidemiology of co occurring addictive and mental disorders: Implications for prevention and service utilization. Am J Orthopsychiatry 1996; 66(1):17-31.

24. McGovern MP, Xie H, Segal SR, Siembab L, Drake RE. Addiction treatment services and co-occurring disorders: Prevalence estimates, treatment practices, and barriers. J Subst Abuse Treat 2006; 31(3):267-275.

25. Stewart AL, Greenfield S, Hays RD, Wells K, Rogers WH, Berry SD, McGlynn EA, Ware JE, Jr. Functional status and well-being of patients with chronic conditions: Results from the Medical Outcomes Study. J Am Med Assoc 1989; 262(7):907-913.

26. Magura S, Staines GL, Blankertz L, Madison EM. The effectiveness of vocational services for substance users in treatment. Subst Use Misuse 2004; 39(13-14):2165-2213.

27. Dunlap LJ, Zarkin GA, Lennox R, Bray JW. Do treatment services for drug users in outpatient drug-free treatment programs affect employment and crime? Subst Use Misuse 2007;42:1161-1185.

28. Gerstein DR, Johnson RA. Nonresponse and selection bias in treatment follow-up studies. Subst Use Misuse 2000; 35(6-8):971-1014. 